\title{
Evaluation of a novel, hybrid model (Mumbai EUS II) for stepwise teaching and training in EUS-guided biliary drainage and rendez- vous procedures $\square$
}

\section{다(1) $(9)$}

\section{Authors}

Vinay Dhir ${ }^{1}$, Takao Itoi ${ }^{2}$, Nonthalee Pausawasdi ${ }^{3}$, Mouen A. Khashab ${ }^{4}$, Manuel Perez-Miranda ${ }^{5}$, Siyu Sun ${ }^{6}$, Do Hyun Park $^{7}$, Takuji Iwashita ${ }^{8}$, Anthony Y. B. Teoh ${ }^{9}$, Amit P. Maydeo ${ }^{10}$, Khek Yu Ho ${ }^{11}$

Institutions

1 Baldota Institute of Digestive Sciences - Endoscopy and Endosonography, Global Hospitals, Parel, Mumbai, India

2 Department of Gastroenterology and Hepatology, Tokyo Medical University, Tokyo, Japan

3 Mahidol University Faculty of Medicine, Siriraj Hospital, Bangkok, Thailand

4 Johns Hopkins Hospital - Gastroenterology, Baltimore, MD, USA

5 Hospital Universitario Rio Hortega - Gastroenterology, Valladolid, Spain

6 Medicine, Sheng Jing Hospital, Liaoning, China

7 Asan Medical Center - Gastroenterology, University of Ulsan, Seoul, Republic of Korea

8 Internal Medicine, Gifu University, Gifu, Japan

9 The Chinese University of Hong Kong - Surgery, Prince of Wales Hospital, Shatin, Hong Kong, China

10 Digestive Diseases Center, Baldota Institute of Digestive Sciences, Global Hospitals, Parel, Mumbai, India

11 Department of Gastroenterology and Hepatology, National University Health System, Singapore, Singapore

submitted 22.4.2017

accepted after revision 10.7.2017

Bibliography

DOI https://doi.org/10.1055/s-0043-118097 |

Endoscopy International Open 2017; 05: E1087-E1095

(C) Georg Thieme Verlag KG Stuttgart · New York

ISSN 2364-3722

Corresponding author

Vinay Dhir, MD, Baldota Institute of Digestive Sciences Endoscopy and Endosonography, Global Hospitals, Parel, Mumbai 400012, India

Fax: +91-22-22804769

vinaydhir@gmail.com

\section{ABSTRACT}

Background and aims EUS-guided biliary drainage (EUSBD) and rendezvous (EUS-RV) are acceptable rescue options for patients with failed endoscopic retrograde cholangiopancreatography (ERCP). However, there are limited training opportunities at most centers owing to low case volumes. The existing models do not replicate the difficulties encountered during EUS-BD. We aimed to develop and validate a model for stepwise learning of EUS-BD and EUS$\mathrm{RV}$, which replicates the actual EUS-BD procedures.

Methods A hybrid model was created utilizing pig esophagus and stomach, with a synthetic duodenum and biliary system. The model was objectively assessed on a grade of $1-4$ by two experts. Twenty-eight trainees were given initial training with didactic lectures and live procedures. This was followed by hands-on training in EUS-BD and EUS-RV on the hybrid model. Trainees were assessed for objective criteria of technical difficulties.

Results Both the experts graded the model as very good or above for all parameters. All trainees could complete the requisite steps of EUS-BD and EUS-RV in a mean time of 11 minutes (8-18 minutes). Thirty-six technical difficulties were noted during the training (wrong scope position, 13; incorrect duct puncture, 12; guidewire related problems, 11). Technical difficulties peaked for EUS-RV, followed by hepaticogastrostomy (HGS) and choledochoduodenostomy (CDS) $(20,9$, and $7, P=0.001)$. At 10 days follow-up, nine of 28 trainees had successfully performed three EUS-RV and seven EUS-BD procedures independently.

Conclusions The Mumbai EUS II hybrid model replicates situations encountered during EUS-RV and EUS-BD. Stepwise mentoring improves the chances of success in EUS-RV and EUS-BD procedures. 


\section{Introduction}

Endoscopic retrograde cholangiopancreatography (ERCP) is the standard option for relief of obstructive jaundice. In recent years, EUS-guided biliary drainage (EUS-BD) and EUS-guided rendezvous (EUS-RV) have emerged as acceptable options in the event of a failed ERCP or where ERCP is not possible [1,2]. However, EUS-BD and EUS-RV are currently required in a small number of patients, and the case volume remains low even at centers with a high volume of biliary interventions $[3,4]$. This creates difficulties in training and teaching under supervision.

Models for EUS-BD are evolving gradually. An ideal model should approximate the human anatomy, should teach both technical and cognitive aspects, and should have good visibility under ultrasound as well as X-ray. It should recreate difficulties encountered during real life situations in human patients. Training on pig models has been attempted, but there are issues with erratic dilation of the biliary system after clipping or ligating the papilla [5]. We have previously described a 3D prototype of the bile duct, which works well for the essential steps of EUS-BD, but it does not have an attached stomach or duodenum, and thus does not simulate real life situations [6]. We have attempted to create a novel hybrid model with a combination of pig stomach and synthetic duodenum and bile duct. In this paper, we describe the creation of the model and the results of stepwise training on this model.

\section{Methods}

This was a prospective observational study on a hybrid model during a course on EUS-BD, and no humans or live animals were used. The institutional ethics committee approved the study.

\section{Creation of Mumbai EUS II hybrid model} The concept

The hybrid model evolved over a 24-month period. The initial plan was to create a replica of human stomach, duodenum, and bile duct, so that exact scope positions and maneuvers could be taught. For this purpose, a three-dimensional computer graphic image of the stomach, duodenum, and bile duct was created using standard measurements of the organs from anatomy textbooks ( $\triangleright$ Fig. 1a). The only exception was the dilated bile duct which measured $1 \mathrm{~cm}$. In the first step, we used this design to create a 3D printed model with polycarbonate, the same material used for our bile duct prototype ( $\downarrow$ Fig. 1b). It had multiple holes at expected puncture sites in the stomach, duodenum, and bile duct. However, this model proved ineffective due to the hardness of polycarbonate, which restricted the echo-endoscope movements. There was no softer material available for $3 \mathrm{D}$ printing and so we decided to abandon 3D printing and shift to rubber. We used the same graphic design to create the model with molded rubber. To maintain the shape of the organs and yet have flexibility, the rubber wall had to be kept $4 \mathrm{~mm}$ thick. We also created a papilla, and a pancreatic duct ( $\triangleright$ Fig.1c). This model allowed free echo-endoscope movements but the transmission of ultrasound waves from the $4 \mathrm{~mm}$ rubber wall of the stomach and duodenum was unsatisfactory, resulting in poor quality images. The poor imaging through the rubber wall gave rise to the idea of using pig stomach. Since the pig bile duct is very small, we decided to create a hybrid model using the stomach from a pig, and with duodenum and bile duct made of molded rubber.

\section{The model ( $\vee$ Fig. 2 )}

The distal $4 \mathrm{~cm}$ esophagus, entire stomach, and duodenum of pig were used after cleaning and disinfection. The greater curvature of the stomach was sutured to create a more tubular stomach to allow easier scope passage. The pig duodenum
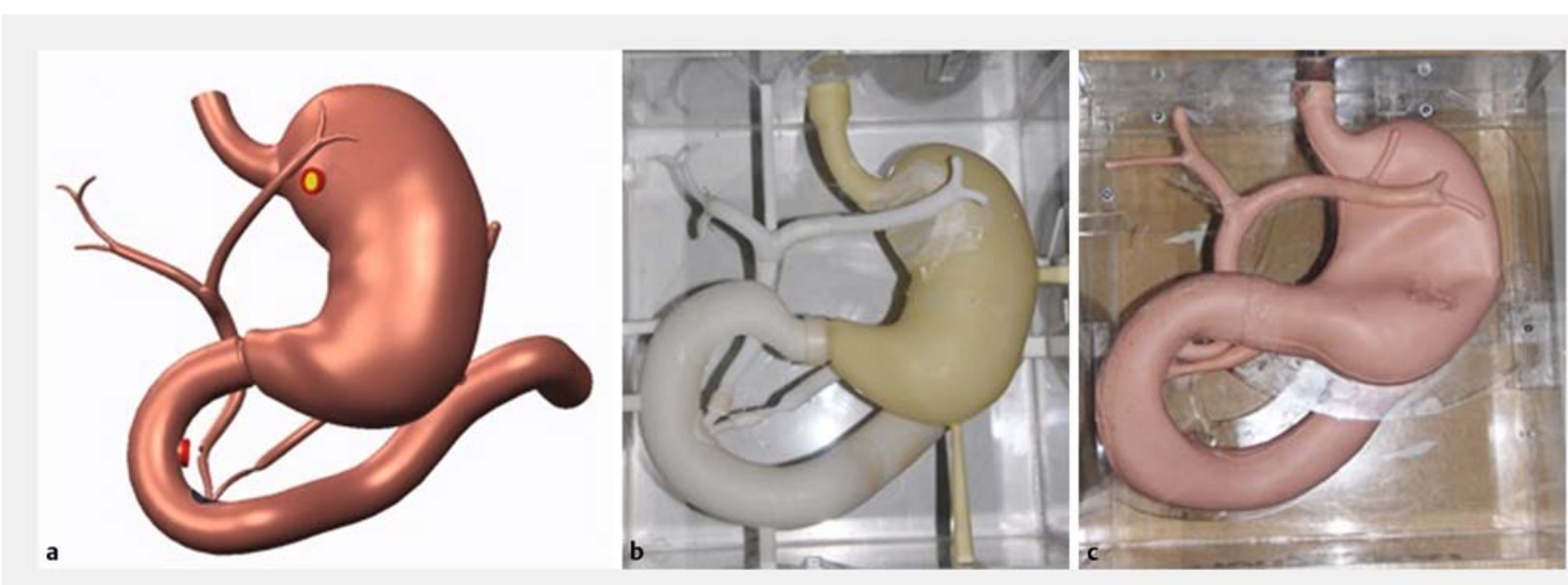

Fig. 1 a Computer graphic image of the model concept. b 3D printed model. c Molded rubber model. Note the holes in the 3D printed model for expected puncture site. 

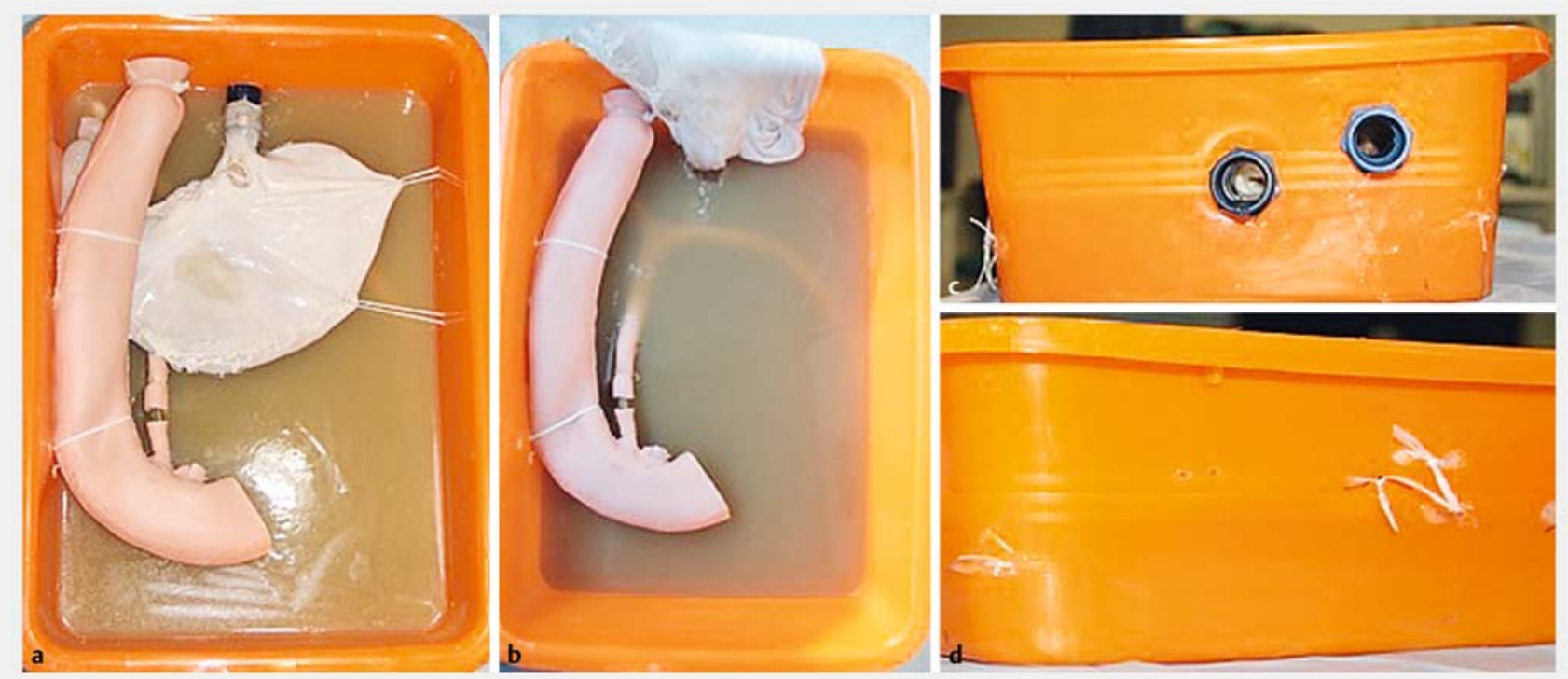

Fig. 2 The Mumbai EUS II hybrid model. a The pig stomach is sutured to create a tubular shape, and anchored to the side of the tray with thread. The duodenum and bile duct are created from molded rubber. A stricture is seen in the distal CBD. $\mathbf{b}$ The pig stomach is lifted up to show the gelatin medium. The proximal bile duct is kept 1 inch under the surface to create a medium between stomach and bile duct. c Separate openings for stomach and duodenum. $\mathbf{d}$ Threads for anchoring stomach and duodenum. Note the threads for duodenum are at a higher level (long side of tray), than those for the bile duct (edge of tray).

was not used for the procedure, but was used for anchoring the stomach in proper position. The duodenum and the bile duct of the molded rubber model created previously were used. The duodenum had a radius of $2 \mathrm{~cm}$, and a papillary opening was created on the medial wall of the second part. The bile duct and the intrahepatic biliary radicals were of $1 \mathrm{~cm}$ radius, and joined the duodenum at the papilla. A stricture was created in the distal bile duct by cutting and re-joining the cut ends together by a stiff and narrow suction hose. This created a stricture with shouldering effect, similar to that of a malignant stricture.

A $17 \times 11 \times 6$ inch plastic tray was used as the base. Gelatin was used as the conducting medium for ultrasound waves. For creation of gelatin medium, $500 \mathrm{~g}$ of gelatin powder was mixed in $5 \mathrm{~L}$ of hot water and mixed until uniform. The mixture was transferred to the tray. The bile duct was then placed about one inch under the surface of the liquid gelatin mix, and anchored in place by a strong thread sutured to the wall of the

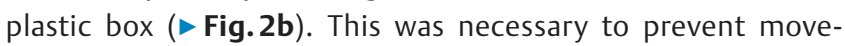
ment of the bile duct away from the desired position during setting of the gelatin. For EUS-RV, the terminal $3 \mathrm{~cm}$ of bile duct was kept above the surface of the gelatin so that it could join the duodenum. For trans-luminal procedures such as hepaticogastrostomy (HCS) and choledochoduodenostomy (CDS), the entire bile duct was kept under the surface of gelatin, as guidewire passage into the duodenum was not needed. The tray was then refrigerated for 3 hours. This allowed the gelatin to solidify and act as a good conducting medium for ultrasound waves.

The pig stomach and the rubber duodenum were anchored on the surface of the gelatin, thus creating a 1 inch thick layer of gelatin between the stomach and the bile duct. The stomach was anchored with the help of thread in such a way that the proximal stomach was over the left bile duct, while the distal stomach was above the duodenum. The pylorus of the pig stomach is very thick and not amenable to needle puncture. The pylorus was anchored to the side of the tray. Thus, both CDS and HGS procedures were actually performed from the stomach itself. The pig esophagus was attached to a port on the narrow side of the tray, while the duodenum was attached to a separate port on the same side of the tray $(\nabla$ Fig. $2 c)$. Thus the stomach and duodenum were not connected, but all the steps of the various procedures could be performed with appropriate scope positions, resembling human anatomy. The material of the bile duct allows dilation with bougie, balloon dilators, or a cystotome (electrocautery). The model was assessed for adequacy of ultrasound and X-ray imaging, feasibility of electrocautery, and wire manipulation, by two experts independently.

\section{Stepwise training}

The schema of stepwise training is shown in $\triangleright$ Fig. 3 . In the second step, the trainees were exposed to technical aspects of various EUS-BD procedures by didactic lectures and live procedures. The hands-on training was divided into three parts over 3 days ( $\bullet$ Fig. 3 , steps $3-5$ ).

On the first day, guidewire manipulation was taught. The Mumbai EUS 3D model was used for this purpose ( $\bullet \mathbf{F i g . 4 , ~}>$ Videos 1 -3). A distal stricture was created by instilling silicone in the distal bile duct, and holes were created in the silicone after it had solidified. The bile duct was fixed on a flat tray and kept under fluoroscopy. The trainees performed predefined tasks 


\begin{tabular}{l} 
Step 1 \\
Choosing the right candidates for training \\
Step 2 \\
Didactic lectures and live demonstrations \\
Step 3 \\
Learning guide wire manipulations \\
\hline Step 4
\end{tabular}

Learning trans-luminal procedures (CDS,HGS)

\section{Step 5}

Learning trans-papillary procedures (EUS-RV, AG)

Fig. 3 The schema of stepwise training.

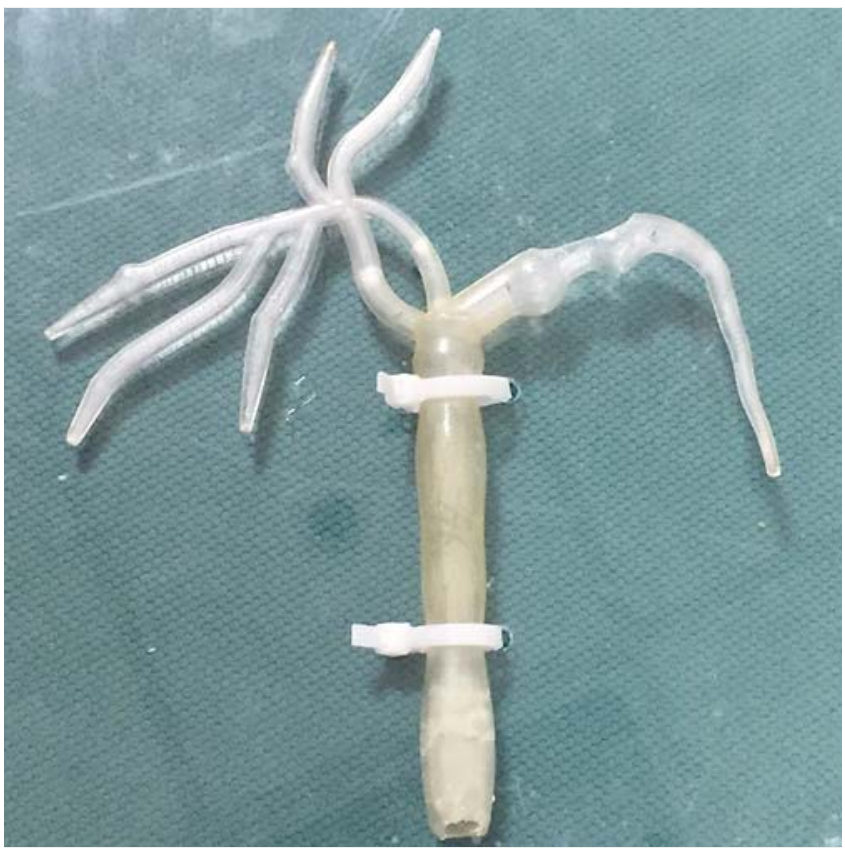

Fig. 4 The Mumbai EUS model used for training in guidewire manipulation.

such as entering the right anterior and right posterior duct from the left duct, and crossing the distal CBD stricture ( $\triangleright \mathbf{V i -}$ deos 1-3).

On the second day, the trainees performed CDS and HGS on the hybrid model. They were taught the appropriate scope position for each procedure, visualization of bile duct, ductal

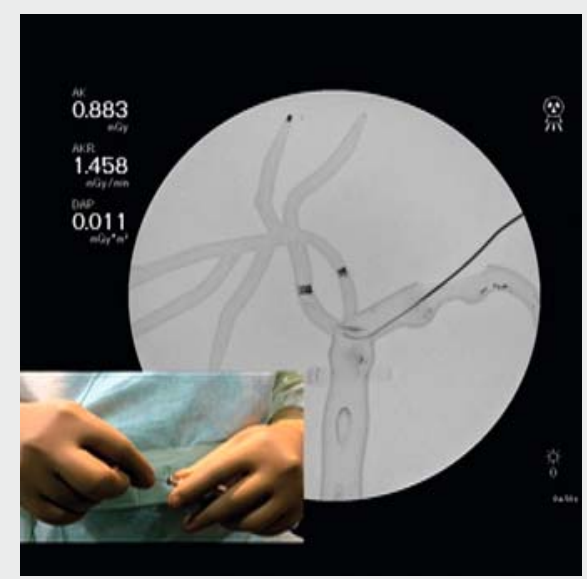

Video 1 Guidewire manipulation across the hilum and distal CBD stricture in the Mumbai EUS model. Note the torque created by hand movements.

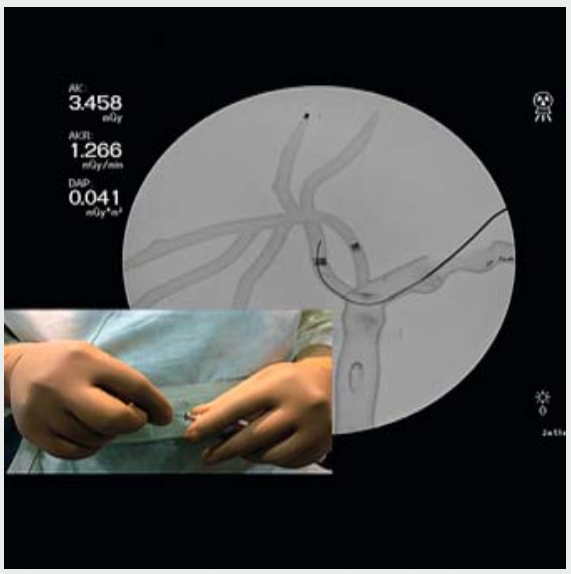

$\nabla$ Video 2 Guidewire manipulation into the right posterior duct in the Mumbai EUS model.

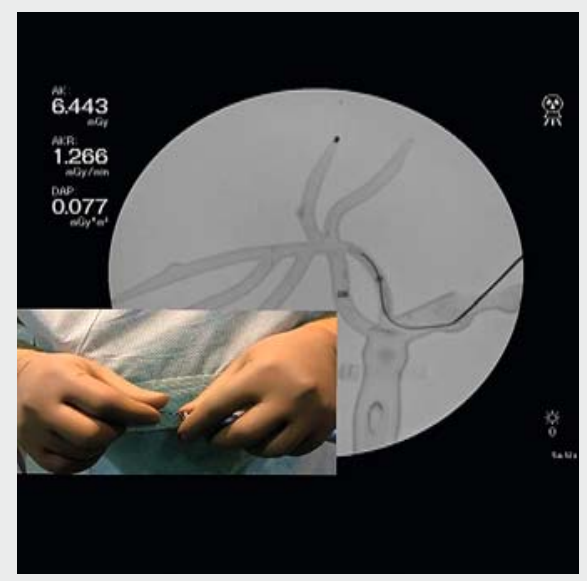

$\nabla$ Video 3 Guidewire manipulation into the right anterior duct in the Mumbai EUS model. 

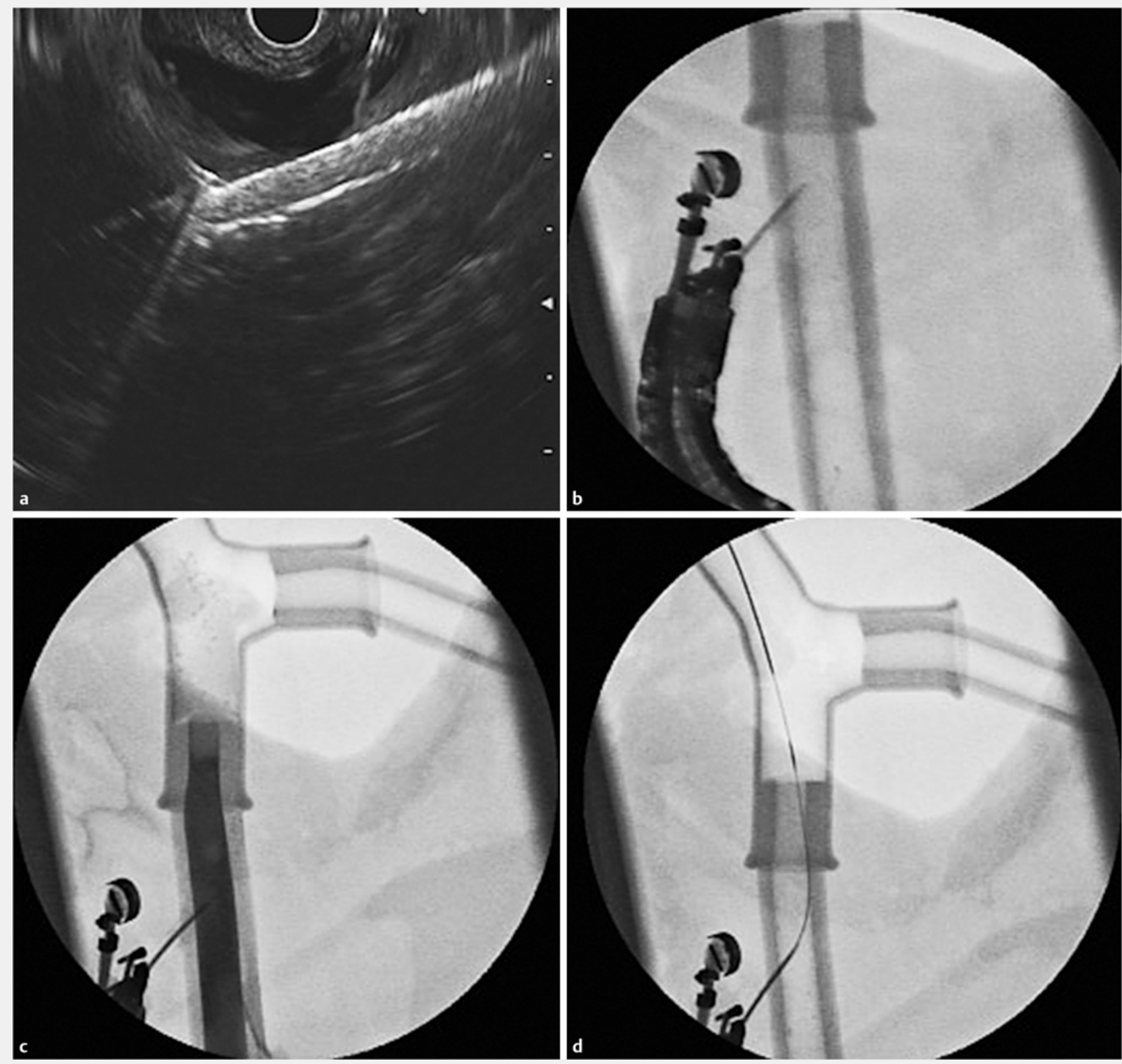

- Fig. 5 CDS in the hybrid model. a CBD puncture under ultrasound guidance. b Echoendoscope position in long loop, looking towards the hilum. This position replicates that required in real patients. c Contrast injection. $\mathbf{d}$ Guidewire passage across the hilum.

puncture with a 19-gauge needle, guidewire manipulation, tract dilation with $6 \mathrm{~F}$ cystotome, and a plastic stent placement ( $\triangleright$ Fig.5). A therapeutic linear echo-endoscope (Olympus TGF140, Olympus Inc, Tokyo, Japan), and a $260 \mathrm{~cm} 0.032$ " or a $400 \mathrm{~cm} \mathrm{0.035"} \mathrm{guidewire} \mathrm{(Terumo,} \mathrm{Tokyo,} \mathrm{Japan,} \mathrm{and} \mathrm{Visiglide,}$ Olympus, Japan) were used along with a $6 \mathrm{~F}$ cystotome (G-Flex, Germany) and plastic stents.

On the third day, they were taught EUS-RV through the trans-hepatic route on the hybrid model. They were taught scope position, needle puncture, and guidewire manipulation across the hilum, the distal stricture, and across the papilla into the duodenum ( $\vee$ Fig.6, $\vee$ Videos 4,5 ). The echo-endoscope was then removed leaving the wire in place, and they in- serted a duodenoscope through the other port into the duodenum. They were taught to catch the wire at the papilla with the snare and pull it up into the duodenoscope channel ( $\vee$ Video 6 ).

\section{Assessment}

The experts graded the various aspects of the model as follows: Grade 1 - average, Grade 2 - good, Grade 3 - very good, and Grade 4 - excellent. Objective assessment of the trainee performance was done. At all stages on all days, they were assisted by an expert who identified their mistakes and corrected them. Performance parameters included correct scope position, needle puncture and visibility under ultrasound, guidewire manipulation and avoidance of shearing, grasping the guidewire in 

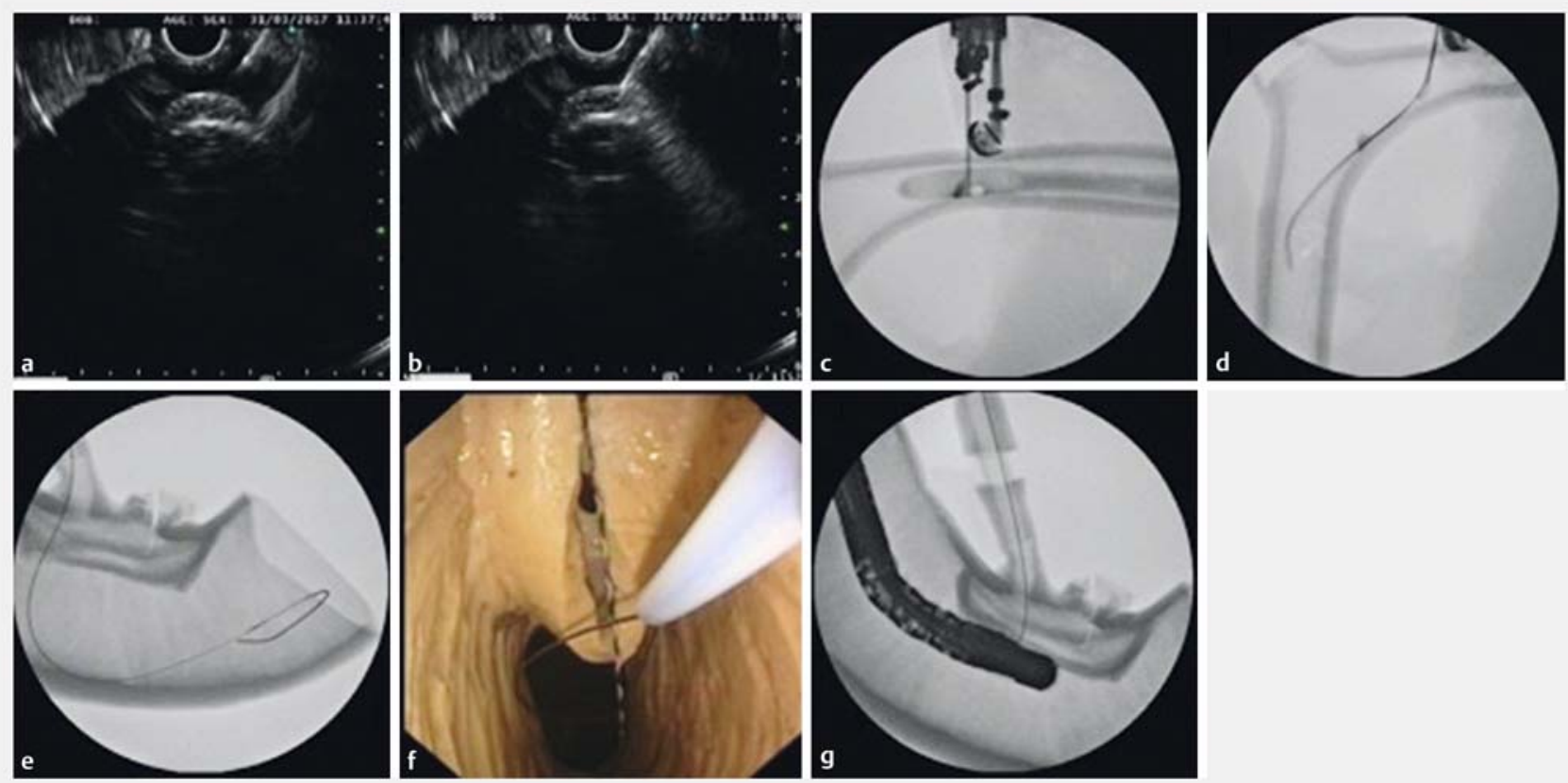

- Fig. 6 EUS-RV in the hybrid model. a The left duct is identified. b Needle puncture. c Contrast injection. $\mathbf{d}$ Guidewire manipulation across the hilum. e Guidewire manipulated across the papilla into the duodenum. $\mathbf{f}$ Endoscopic image showing a snare grasping the guidewire. $\mathbf{g}$ Duodenoscope in front of the papilla with guidewire secured.

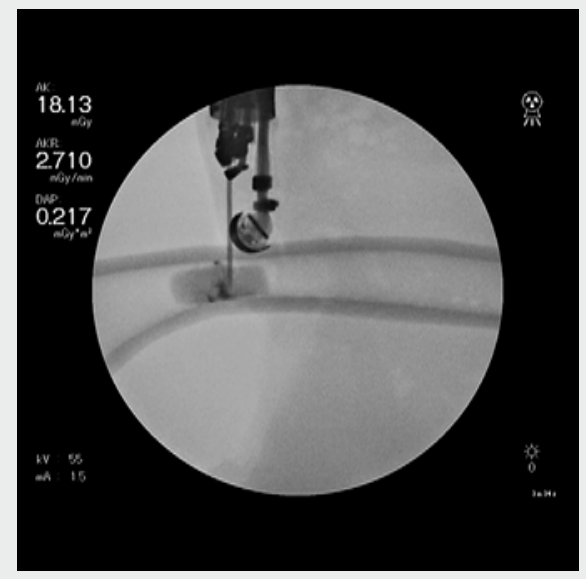

Video 4 Identification of left duct, duct puncture with a 19 gauge needle, and contrast injection in the hybrid Mumbai EUS II model.

the duodenum, and pulling the guidewire into duodenoscope biopsy channel. Deficiencies for each trainee were noted and corrected.

\section{Results}

All of the trainees had experience of EUS and EUS-FNA for a minimum of 2 years ( $2-10$ years). A total of 18 hybrid models were used. The bile duct could be clearly visualized for CDS,

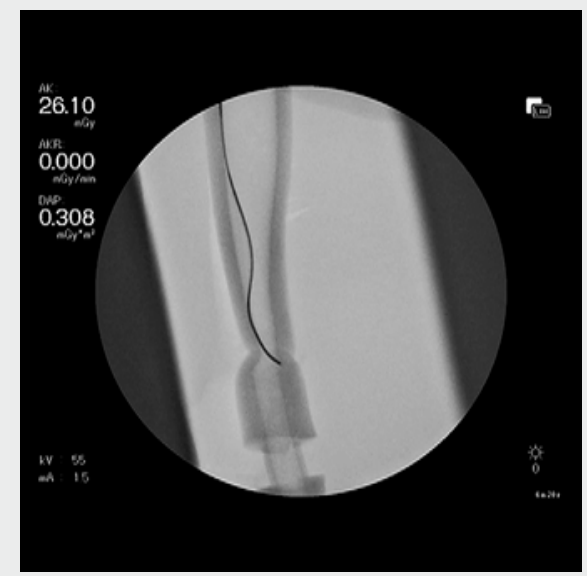

$\checkmark$ Video 5 Wire manipulation across the hilum, CBD, and papilla in the duodenum (Mumbai EUS II model).

HGS, and EUS-RV in all of the models apart from one. The needle could be visualized entering the bile duct in all of the models. It was possible to use electrocautery and $6 \mathrm{~F}$ cystotome in all of the models. The bile duct created a minimal shadowing effect on X-ray, which did not interfere with the procedure. One tray could be used for a mean of four training sessions (range, 1-7). The objective assessment of the model by experts is shown in Table 1. All of the trainees could finish the guidewire manipulation exercises within the stipulated time of 15 
- Table 1 Objective assessment of the model.

\begin{tabular}{|l|l|l|l|}
\hline & Expert 1 & Expert 2 & $\begin{array}{l}\text { Average } \\
\text { score }\end{array}$ \\
\hline Scope position HGS & 3 & 4 & 3.5 \\
\hline Scope position CDS & 3 & 3 & 3 \\
\hline Needle visibility (EUS) & 4 & 4 & 4 \\
\hline Duct visibility(EUS) & 3 & 3 & 3 \\
\hline Duct visibility (X-ray) & 4 & 4 & 4 \\
\hline Guidewire manipulation & 4 & 3 & 3.5 \\
\hline Guidewire retrieval (RV) & 4 & 3 & 3.5 \\
\hline Cautery usage & 4 & 4 & 4 \\
\hline Stent placement & 4 & 4 & 4 (R) \\
\hline $\begin{array}{l}\text { CDS, choledochoduodenostomy; HGS, hepaticogastrostomy; RV, EUS-guid- } \\
\text { ed rendezvous. }\end{array}$ & & & \\
\hline
\end{tabular}

minutes on day 1 . All of the trainees completed all of the procedures apart from one CDS where the scope could not be well positioned as the stomach sutures did not allow appropriate scope position. The procedure could be completed with a fresh model. There were no failures because experts were assisting them to complete the procedure. A total of 36 technical deficiencies were noted during trainee performance ( $\triangleright$ Table 2 ). Most of the difficulties were due to inappropriate scope position or difficulties in wire manipulation. There were more difficulties encountered during EUS-RV compared to CDS (20 vs. 7, $P=0.001)$ and HGS (20 vs. 9, $P=0.006$ ). No candidate had a problem with tract dilation or stent placement. At 10 days post-training, nine trainees had reported performing three EUS-RV and seven EUS-BD procedures independently at their respective institutes.

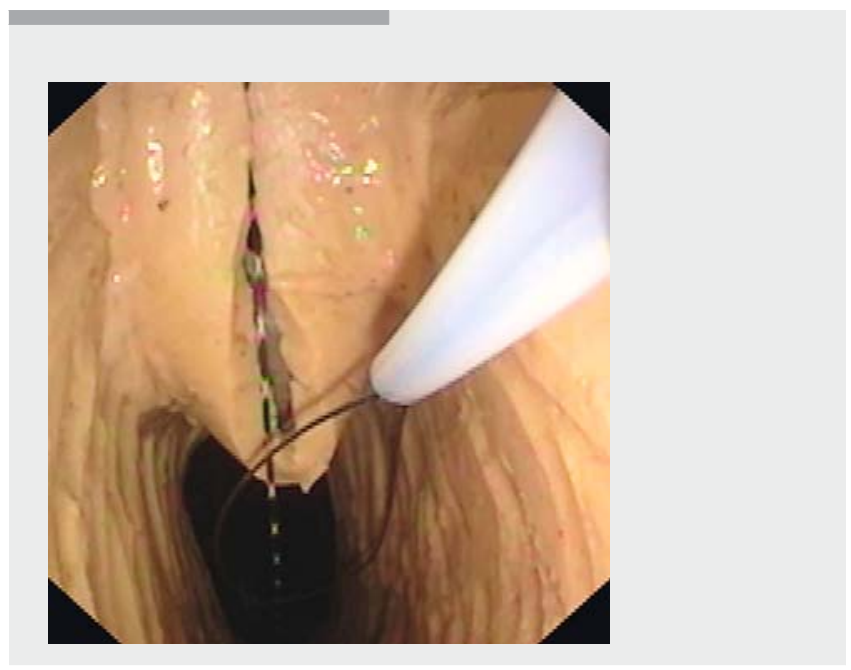

Video 6 Duodenoscopic view of the duodenum and papilla, grasping of the guidewire at the papilla with the snare, and pulling the wire into the duodenoscope channel (Mumbai EUS II model).

\section{Discussion}

Recent studies have shown EUS-BD to be superior to percutaneous biliary drainage in patients with failed ERCP $[7,8]$. The utilization of EUS-BD is likely to increase in the coming years. The biggest bottleneck for growth of EUS-BD appears to be lack of training opportunities. The number of EUS-BD cases is small even at specialized high volume units. We have previously published our experiences with a 3D printing model, which appears suitable for teaching the essential basics of EUS-BD [6]. However, EUS-BD is a conglomerate of multiple procedures with different access points, each having its own unique technique. Thus, a model is required which goes beyond the basic steps, and is able to demonstrate the unique nature of each of these procedures. Optimum visibility under X-ray and ultrasound is the first requirement as all stent placements require $X$-ray, at least for now. As with ERCP, a good scope position is another must for a successful EUS-BD procedure. Moreover, visibility of the needle and then the guidewire under ultrasound is required continuously throughout the procedure. All of these aspects can only be integrated, if the anatomy of the model approximates human anatomy. Our earlier 3D model lacked a stomach and duodenum, making it difficult to learn optimum scope positions. Thus, we endeavored to improve the 3D model by incorporating a stomach and duodenum in it. However, we could not find a suitable material for 3D printing, which imitated the flexibility of the gut wall. We experimented with molded rubber, but ultrasound penetration was not optimum through the rubber stomach wall. We found the pig stomach to be ideal for this purpose. However, the pig duodenum was not suitable as the papilla is higher up near the duodenal bulb, and the bile duct is very thin. Thus, we decided to create a hybrid model with pig stomach and rubber duodenum and bile duct.

Gelatin has been used for many years for training in abdominal ultrasound. It allows excellent transmission of ultrasound waves, and has a tissue like appearance under ultrasound [9]. Hence, we chose to use a gelatin interface between the bile duct and the stomach and duodenum. The interface was created by suspending the bile duct in liquid gelatin before it solidified, and holding it in position with a strong thread. Once the gelatin solidifies, the duct remains in position. If appropriately frozen, gelatin retains its character for about $48 \mathrm{~h}$ before disintegrating. This period is sufficient for most training situations. Since it is very difficult to connect a pig stomach to a rubber duodenum, we used separate ports for entry into the stomach and duodenum. While this disturbed the anatomy, it did not interfere with the demonstration of correct techniques. We could teach the correct technique and scope positioning for CDS from the pig stomach by anchoring the distal stomach in front of the bile duct.

We chose our trainees carefully. The trainees had a minimum 2 years of independent experience of EUS and EUS-FNA. There are no published guidelines on who is well suited to perform EUS-BD. The Asian EUS Group guidelines (Teoh et al., unpublished data) stipulate expertise in ERCP, EUS, and EUS-FNA as desirable for performing EUS-BD. EUS-BD involves complex 
- Table 2 Technical difficulties during trainee performance.

\begin{tabular}{|l|l|l|l|}
\hline & CDS (n=28) & HGS (n=28) & RV (n=28) \\
\hline Wrong scope position & 4 & 5 & 4 \\
\hline Incorrect duct puncture & 2 & 2 & 8 \\
\hline Guidewire passage in wrong direction & 0 & 1 & 1 \\
\hline Guidewire shearing & 1 & 1 & 1 \\
\hline Wire stuck in needle & & 0 & 1 \\
\hline Guidewire manipulation at stricture & 0 & 0 & 1 \\
\hline Guidewire retrieval in duodenum & 0 & 0 & 1 \\
\hline Guidewire slippage during retrieval & 0 & 0 & $9(32.14 \%)$ \\
\hline Total & 7 (25\%) & 20 (71.42\%) \\
\hline CDS, choledochoduodenostomy; HGS, hepaticogastrostomy; RV, EUS-guided rendezvous. & & 1 \\
\hline
\end{tabular}

trans-luminal techniques, and it is imperative that those performing it have the requisite expertise in scope and accessory handling. The follow-up of our training reflects that our trainees were ready for EUS-BD. Nine of them performed 10 procedures successfully within 10 days of training.

We designed our training keeping in mind the problems encountered during EUS-BD. Most of the procedures fail in the initial phase for two reasons $[10,11]$. First, the inability to find a good scope position to puncture, and hold this position securely during the procedure. Second, the inability to manipulate the guidewire across a stricture and even normal areas such as liver hilum and papilla. Thus, the trainees were exposed to live procedures from experts in the beginning, with explanation of key points. Then the first step of learning involved guidewire manipulation. The trainees targeted specific ducts, and were taught how to reach them. The next phase involved trans-luminal procedures. The latter do not depend so much on guidewire manipulation, and are considered easier, as demonstrated by the higher published success rates of trans-luminal procedures compared to trans-papillary or antegrade procedures [1]. Thus, they learned scope positioning, identification of puncture site, importance of needle and guidewire visibility during the procedure, tract dilation, and stent placement. Finally, they were exposed to the antegrade transpapillary procedures. They learned guidewire manipulation and maintenance across the papilla, and retrieval of guidewire from the duodenum into the duodenoscope channel. There was an expert assisting them with their difficulties at all points during the training, and every day, the trainees in groups of 3-4 discussed their problems with an expert over 30 minutes. Thus, a stepwise approach helped them acquire the requisite expertise in different aspects of different EUS-BD procedures.

There were certain limitations in this study. First, we did not use metal stents. Correct placement of metal stents during trans-luminal procedures is an essential learning point. However, this was not done to preserve the model for repeat use, as placement of a metal stent would have meant one model per trainee per procedure. Second, the trainees were carefully pre-selected. We do not know if the hybrid model and the stepwise approach would work as well with less experienced endosonographers. Third, the model does not have a communication between the stomach and duodenum. However, this did not inhibit the performance of various procedures, except that the CDS procedure was actually taught from the distal stomach. Fourth, we require $X$-rays for training on this model, thus limiting the utility of this model for out-of-hospital training. However, we believe that X-ray training is essential for learning appropriate guidewire manipulation and other steps of EUS-BD. It is possible that X-rays may not be needed when hot stents are used. Improvements in 3D materials could also allow creation of a 3D printed model of the stomach, duodenum, and bile duct, with properties similar to human tissue.

In conclusion, we have shown that the Mumbai EUS II hybrid model simulates real life situations encountered during various EUS-BD procedures, and results in excellent learning outcomes, if combined with a stepwise learning process.

\section{Acknowledgements}

We thank the Asian EUS Group for funding construction of the model, and Mr Sehajad Vora and Mr Ganesh Shirodkar for technical assistance.

\section{Competing interests}

None

\section{References}

[1] Dhir V, Isayama H, Itoi T et al. EUS-guided biliary and pancreatic duct interventions. Dig Endosc 2017; 29: $472-485$

[2] Hara K, Yamao K, Mizuno N et al. Endoscopic ultrasonography-guided biliary drainage: Who, when, which, and how? World J Gastroenterol 2016; 22: $1297-1303$ 
[3] Holt BA, Hawes R, Hasan M et al. Biliary drainage: role of EUS guidance. Gastrointest Endosc 2016; 83: 160 - 165

[4] Tonozuka R, Itoi T, Tsuchiya T et al. EUS-guided biliary drainage is infrequently used even in high-volume centers of interventional EUS. Gastrointest Endosc 2016; 84: 206-207

[5] Alcaide N, Lorenzo-Pelayo S, Ruiz-Zorrilla R et al. Endoscopic porcine model of biliary obstruction using over-the-scope clips: Feasibility and applicability to training in EUS-guided drainage procedures. Gastrointest Endosc 2013; 77: AB294-AB295

[6] Dhir $\mathrm{V}$, Itoi T, Fockens $\mathrm{P}$ et al. Novel ex vivo model for hands-on teaching of and training in EUS-guided biliary drainage: creation of "Mumbai EUS" stereolithography/3D printing bile duct prototype (with videos). Gastrointest Endosc 2015; 81: 440-446

[7] Artifon EL, Aparachio D, Paione JB et al. Biliary drainage in patients with unresectable malignant obstruction where ERCP fails: endoscopic ultrasonography-guided versus percutaneous drainage. J Clin Gastroenterol 2012; 46: 768 - 774
[8] Lee TH, Choi JH, Park DH et al. Similar efficacies of endoscopic ultrasound-guided transmural and percutaneous drainage for malignant distal biliary obstruction. Clin Gastroenterol Hepatol 2016; 14: 1011 1019

[9] Fornage B. A simple phantom for training in ultrasound-guided needle biopsy using the freehand technique. J Ultrasound Med 1989; 8: 701

[10] Dhir V, Itoi T, Khashab MA et al. Multicenter comparative evaluation of endoscopic placement of expandable metal stents for malignant distal common bile duct obstruction by ERCP or EUS-guided approach. Gastrointest Endosc 2015; 81: 913 - 923

[11] Sharaiha RZ, Kumta NA, Desai AP et al. Endoscopic ultrasound guided biliary drainage versus percutaneous transhepatic biliary drainage: predictors of successful outcome in patients who fail endoscopic retrograde cholangiopancreatography. Surg Endosc 2016; 30: 5500 5505 